\title{
Combinative evaluation of primary tumor and lymph nodes to predict pelvic lymphatic metastasis in cervical cancer: an integrated PET-IVIM MRI study
}

\author{
Chen $\mathrm{Xu}^{1,2}$, Xiaoran $\mathrm{Li}^{1}$, Yanchi Shi ${ }^{1}$, Bo Wang ${ }^{1}$ and Hongzan Sun ${ }^{1,2^{*}}$
}

\begin{abstract}
Background: The aim of this study was to evaluate the value of combining pelvic lymph node and tumor characteristics on positron emission tomography-intravoxel incoherent motion magnetic resonance (PET-IVIM MR) imaging for predicting lymph node metastasis in patients with cervical cancer, especially in those with negative lymph nodes on PET.

Methods: The medical records of 95 patients with cervical cancer who underwent surgical resection with pelvic lymph node dissection were evaluated. The patients were divided into negative and positive groups according to postoperative pathologic lymph node diagnosis, and comparisons of the PET and IVIM-derived parameters between the two groups were performed. Univariate and multivariate analyses were performed to construct a predictive model of lymph node metastasis.

Results: For all patients, tumor SUV $\max _{1} T L G, D_{\min }$, PET and MRI for lymph node diagnosis showed significant differences between patients with and without confirmed lymph node metastasis. Univariate and multivariate logistic analysis showed that the combination of tumor TLG, $D_{\text {min }}$ and PET for lymph node diagnosis had the strongest predictive value (AUC 0.913, $p<0.001$ ). For patients with PET-negative lymph nodes, SUV max, $S U V_{\text {mean }}$, $M T V, T L G$, and $D_{\min }$ showed significant between-group differences, and univariate and multivariate logistic analysis showed that TLG had the strongest predictive value.
\end{abstract}

Conclusions: The combination of tumorTLG, $D_{\min }$ and PET for lymph node diagnosis is a powerful prognostic factor for all patients. TLG has the best predictive performance in patients with PET negative lymph nodes.

Keywords: Cervical cancer, Positron-emission tomography, Diffusion magnetic resonance imaging, Lymph node

\footnotetext{
* Correspondence: sunhongzan@126.com

'Department of Radiology, Shengjing Hospital of China Medical University, Sanhao Street No36, Heping District, Shenyang, Liaoning, PR China 110004 ${ }^{2}$ Liaoning Provincial Key Laboratory of Medical Imaging, Sanhao Street No36, Heping District, Shenyang 110004, Liaoning, China
}

C C The Author(s). 2020 Open Access This article is licensed under a Creative Commons Attribution 4.0 International License, which permits use, sharing, adaptation, distribution and reproduction in any medium or format, as long as you give appropriate credit to the original author(s) and the source, provide a link to the Creative Commons licence, and indicate if changes were made. The images or other third party material in this article are included in the article's Creative Commons. licence, unless indicated otherwise in a credit line to the material. If material is not included in the article's Creative Commons licence and your intended use is not permitted by statutory regulation or exceeds the permitted use, you will need to obtain permission directly from the copyright holder. To view a copy of this licence, visit http://creativecommons.org/licenses/by/4.0/. The Creative Commons Public Domain Dedication waiver (http://creativecommons.org/publicdomain/zero/1.0/) applies to the data made available in this article, unless otherwise stated in a credit line to the data. 


\section{Background}

Cervical cancer, one of the common malignant tumors of the female reproductive system, is a serious threat to women's health and life [1]. Local recurrence and distant metastasis are the main causes of death. At present, treatment and prognosis plans for patients with cervical cancer are mainly based on the Federation International of Gynecology and Obstetrics (FIGO) stage. According to FIGO 2018 for cervical cancer, regardless of tumor size and parametrial infiltration, the involvement of lymph node metastasis is classified as stage IIIC. Therefore, accurate diagnosis of lymphatic metastasis is crucial for developing individualized treatment plans, improving prognosis, and reducing mortality [2-4].

Recently, fluoro-D-glucose (FDG)-positron emission tomography (PET) has been applied to the diagnosis of cervical cancer metastatic lymph nodes. Rather than computed tomography (CT) or magnetic resonance imaging (MRI), which identifies lymphatic metastasis according to short diameter length and morphology [5].FDG-PET provides quantified metabolic information about lymph nodes and is widely used in the evaluation of lymph node metastasis [6]. One study has proved the prognostic value of lymph node metabolism information in patients with cervical cancer [7]. A lymph node with significantly higher FDG metabolism than the background level is defined as a PET-positive lymph node [8, 9]. However, FDG is not a specific imaging agent, and lymph node reactive hyperplasia is also characterized by high metabolism resulting in many false positive cases. Also, the limited resolution of PET and partial volume effect will also affect the diagnostic accuracy of small lymph nodes.

Therefore, quantitative analysis of lymph nodes alone is ambiguous to achieve satisfied accuracy for predicting lymph node metastasis. In this study, we present a combined model (PET and intravoxel incoherent motion (IVIM)-derived imaging of primary tumors and PET/ MRI diagnosis of lymphatic metastasis) for predicting lymphatic metastasis confirmed by postoperative pathology in all patients and patients with PET-negative lymph nodes.

\section{Methods}

\section{Patients}

We retrospectively collected the medical records of patients with a high suspicion of cervical cancer who had underwent PET-IVIM MRI in our hospital from April 2017 to September 2019. The study participants met the following inclusion criteria: (1) underwent surgery; (2) did not receive radiotherapy or chemotherapy before surgery; and (3) had confirmed cervical cancer via postoperative pathology.

\section{Pathologic diagnosis}

Postoperatively, the tissue was transported to the pathology department of our hospital. Hematoxylineosin (HE) stained sections were evaluated by two pathologists with more than 10 years of experience. The following information was recorded: lymph node metastasis, histologic tumor type, cervical stromal invasion depth, and tumor differentiation grade. The patient with one or more pelvic metastatic lymph nodes identified with pathological results was regarded as a positive case.

\section{PET-MRI scanning and image acquisition}

All patients underwent GE Signa integrated PET/MRI (Signa, GE Healthcare), which combined a $3.0 \mathrm{~T}$ MRI scan (GE Signa 750w) and TOF-PET, allowing simultaneous collection of both PET and MRI data. Before the examination, the patients fasted for 4 to $6 \mathrm{~h}$ with measured blood glucose levels lower than $7.0 \mathrm{mmol} / \mathrm{L} .{ }^{18} \mathrm{~F}$ FDG $(4 \mathrm{MBq} / \mathrm{kg})$ was injected through the cubital vein in the resting state, and PET/MRI was performed after $60 \pm 12 \mathrm{~min}$ with a 32-channel coil (Upper Anterior Array, UAA). For PET scanning execution, a Dixon MRI sequence was used to attenuate the gamma rays, and LIST-mode and the ordered subset maximum expected iteration method were used to reconstruct the images. The MR-IVIM imaging parameters are summarized in Table 1. PET/MRI acquisition sequence and durations are shown in Fig. 1.

\section{Image analysis}

Image analysis using AW4.6 (GE Medical System) workstation was performed by 2 radiologists with more than 4 years of radiodiagnostic experience. After delineation of a lesion region of interest (ROI) at the optimal level of the PET image, the fused-PET/MRI software automatically calculated the metabolic tumor volume (MTV), total lesion glycolysis (TLG), and maximum and mean standardized uptake values $\left(\mathrm{SUV}_{\max }\right.$ and $\left.\mathrm{SUV}_{\text {mean }}\right)$ of the entire tumor. A $40 \% \mathrm{SUV}_{\max }$ threshold was used to calculate MTV $[10,11]$. The IVIM data were analyzed using IMAgenGINE MRToolbox software (Vusion Tech Ltd). The IVIM formula was: $\mathrm{Sb} / \mathrm{S} 0=\mathrm{F}$ exp. $\left[-\mathrm{b} \times\left(\mathrm{D}^{*}+\right.\right.$ $\mathrm{D})]+(1-\mathrm{F}) \times \exp -(\mathrm{b} \times \mathrm{D})$ [12]. On the axial T2-weighted image, the radiologists delineated ROIs at all levels of the lesion, and then the software automatically generated the volume of interest (VOIs) and copied them to the apparent diffusion coefficient (ADC) map, diffusionrelated coefficient (D) map, perfusion related diffusion coefficient $\left(D^{*}\right)$ map, and perfusion-related parameter (f) map, obtaining the parameters $\mathrm{ADC}_{\text {mean }} \mathrm{ADC}_{\text {min }}$, $\mathrm{D}_{\text {mean }}, \mathrm{D}_{\text {min }}, \mathrm{D}^{*}$, and $\mathrm{f}$. 
Table 1 Parameters used for MR-IVIM imaging

\begin{tabular}{llllll}
\hline & Axial T1 & Axial T2 & Sagittal T2 & DWI & Axial IVIM \\
\hline TR & $500 \mathrm{~ms}$ & $498 \mathrm{~ms}$ & $4323 \mathrm{~ms}$ & $4000 \mathrm{~ms}$ & $6900 \mathrm{~ms}$ \\
TE & $8 \mathrm{~ms}$ & $79 \mathrm{~ms}$ & $65 \mathrm{~ms}$ & $238 \mathrm{~ms}$ & minimum \\
Thickness & $6.0 \mathrm{~mm}$ & $6.0 \mathrm{~mm}$ & $6.0 \mathrm{~mm}$ & $6.0 \mathrm{~mm}$ & $8.0 \mathrm{~mm}$ \\
Interval & $2.0 \mathrm{~mm}$ & $2.0 \mathrm{~mm}$ & $1.2 \mathrm{~mm}$ & $2.0 \mathrm{~mm}$ & $9.0 \mathrm{~mm}$ \\
FOV & $26 \mathrm{~cm}$ & $36 \mathrm{~cm}$ & $24 \mathrm{~cm}$ & $40 \mathrm{~cm}$ & $40 \mathrm{~cm}$ \\
Matrix size & $384 \times 384$ & $384 \times 384$ & $384 \times 384$ & $128 \times 128$ & $128 \times 128$ \\
NEX & 2 & 1.5 & 4 & 6 & 6 \\
b-values $\left(\mathrm{s} / \mathrm{mm}^{2}\right)$ & & & & 0,800 & $0,10,25,50,75,100,125,150,200,300,400,600,800$, and 1000
\end{tabular}

TR Repetition time, TE Echo time, FOV Field of view, NEX Number of excitations

\section{PET and MRI diagnosis of lymphatic metastasis}

By comparing with surrounding background tissues, a lymph node with increased uptake by visual assessment is considered as a PET-positive lymph node, independently of node size. The method of PET image analysis was based on the previous literatures $[8,9]$. Patients with one or more positive lymph nodes on PET imaging were considered to be patients with PET positive lymph nodes. Lymph nodes with short axis diameter greater than $1.0 \mathrm{~cm}$ and morphological characteristics on MRI were considered as MRI-positive lymph nodes [13, 14]. The results were independently evaluated by two radiologists with 12 and 13 years of experience in nuclear medicine and radiology.

\section{Statistical analysis}

The data were analyzed using MedCalc software (version 15.2.2), and a $p$ value less than 0.05 was considered as statistical significance. The patients were divided into negative and positive group according to postoperative pathological lymph node diagnosis. Differences in lymph node diagnosis by PET/MRI between groups were evaluated using the chi-square test. The values of PET- and IVIM-derived parameters of the primary tumor were used for the quantitative statistical analyses and the between-groups comparison was performed using the ttest or Mann-Whitney U test. Interclass correlation coefficient (ICC) was used to determine the consistency of the results recorded by the two radiologists $(0.8-1$ indicated high consistency) [15]. Prediction models for pelvic lymph node metastasis were constructed by univariate and multivariate logistic regression (variables with $p<0.1$ in the univariate analysis were used in the multivariate analysis. Bonferroni corrections were performed to correct multivariate analysis). Receiver operating characteristic (ROC) curves were generated for
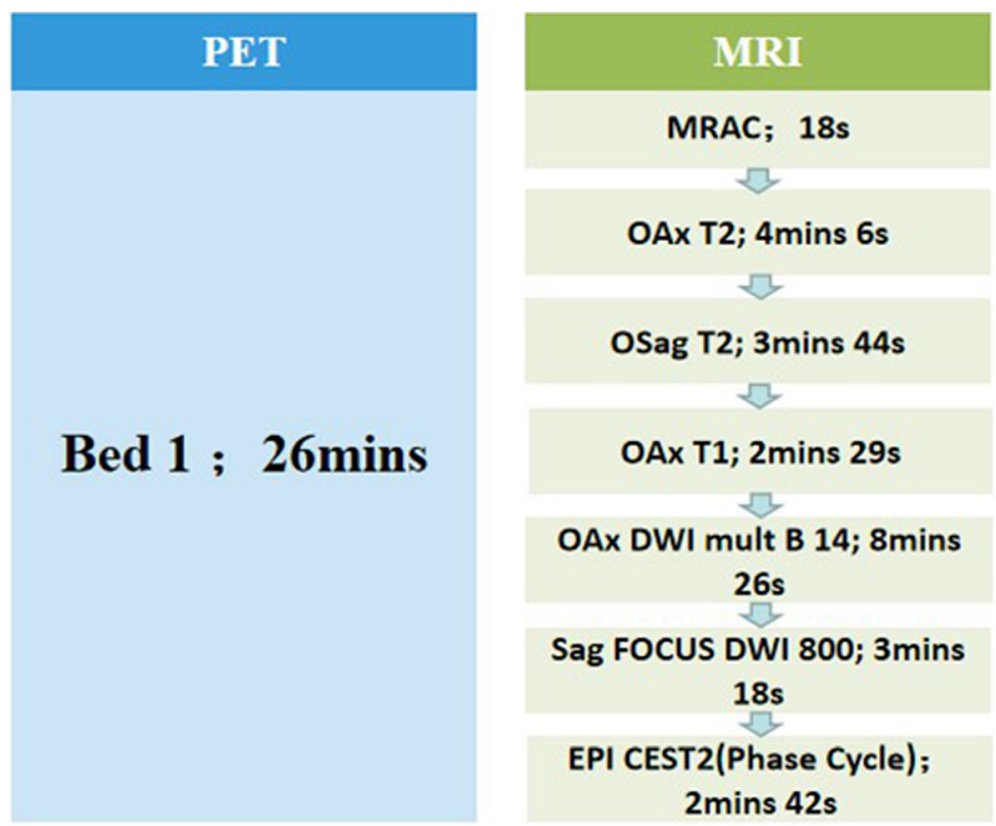

Fig. 1 PET and MRI scan at the same time, each sequence of MRI is performed in sequence, the total time is about 26 min 
combined or individual parameters to assess the area under the ROC curve (AUC) for differentiating the pelvic lymph node status and obtaining the cut-off threshold values corresponding to the highest value of the Jordan index. Comparison between the ROC curves was performed with the DeLong test. The correlation between parameters was analyzed by Spearman rank correlation.

\section{Results}

\section{Patient characteristics}

Ninety-five patients were enrolled (Fig. 2): 78 without lymph node metastasis and 17 with lymph node metastasis (Figs. 3 \& 4). The clinicopathological characteristics of the participants are summarized in Table 2.

\section{ICC statistics}

Interobserver agreements were excellent for tumor SUVmax $\mathrm{SUV}_{\text {mean }}, \mathrm{MTV}, \mathrm{TLG}, \mathrm{ADC}_{\text {mean }}, \mathrm{ADC}_{\text {min }}, \mathrm{D}_{\text {mean }}$, $\mathrm{D}_{\text {min }}, \mathrm{D}^{\prime \prime}$, and $\mathrm{f}$ value (ICC,0.992, 0.991, 0.993, 0.993, $0.862,0.822,0.842,0.811,0.822$ and 0.801 , respectively).
The relationship between lymph node metastasis and imaging parameters in all patients

In all patients, $\operatorname{SUV}_{\text {max }}(p=0.048)$, TLG $(p=0.014)$, $\mathrm{D}_{\text {min }}$ $(p=0.020)$, MRI in lymph node diagnosis $(p=0.009)$ and PET in lymph node diagnosis $(p<0.001)$ showed significant differences between patients with and without lymph node metastasis (Table 3 ). The remaining PETand IVIM-derived parameters did not show statistical differences between the two groups.

ROC analysis of the negative and positive groups according to postoperative lymph node diagnosis showed that $\mathrm{SUV}_{\text {max }}$ (AUC 0.654, $p=0.023$ ), $\mathrm{SUV}_{\text {mean }}$ (AUC 0.646, $p=$ 0.030 ), TLG (AUC 0.692, $p=0.009$ ), $\mathrm{D}_{\min }$ (AUC 0.681, $p=$ 0.007 ), MRI in lymph node diagnosis (AUC $0.656, p=0.020$ ) and PET in lymph node diagnosis (AUC 0.736, $p<0.001$ ) had a positive effect on predicting metastatic lymph nodes confirmed by postoperative pathology (Fig. 4). The optimal cut-off threshold values for $\mathrm{SUV}_{\max }, \mathrm{SUV}_{\text {mean }}$, TLG, and $D_{\min }$ were $12.98 \mathrm{~g} / \mathrm{cm}^{3}$ (sensitivity 76.47 , specificity 53.85 ), $8.61 \mathrm{~g} / \mathrm{cm}^{3}$ (sensitivity 70.59 , specificity 60.26 ), $168.7 \mathrm{~g}$ (sensitivity 52.94, specificity 82.05 ), and $0.55 \times 10^{-3} \mathrm{~mm}^{2} / \mathrm{s}$ (sensitivity 82.35 , specificity 56.41 ) respectively.

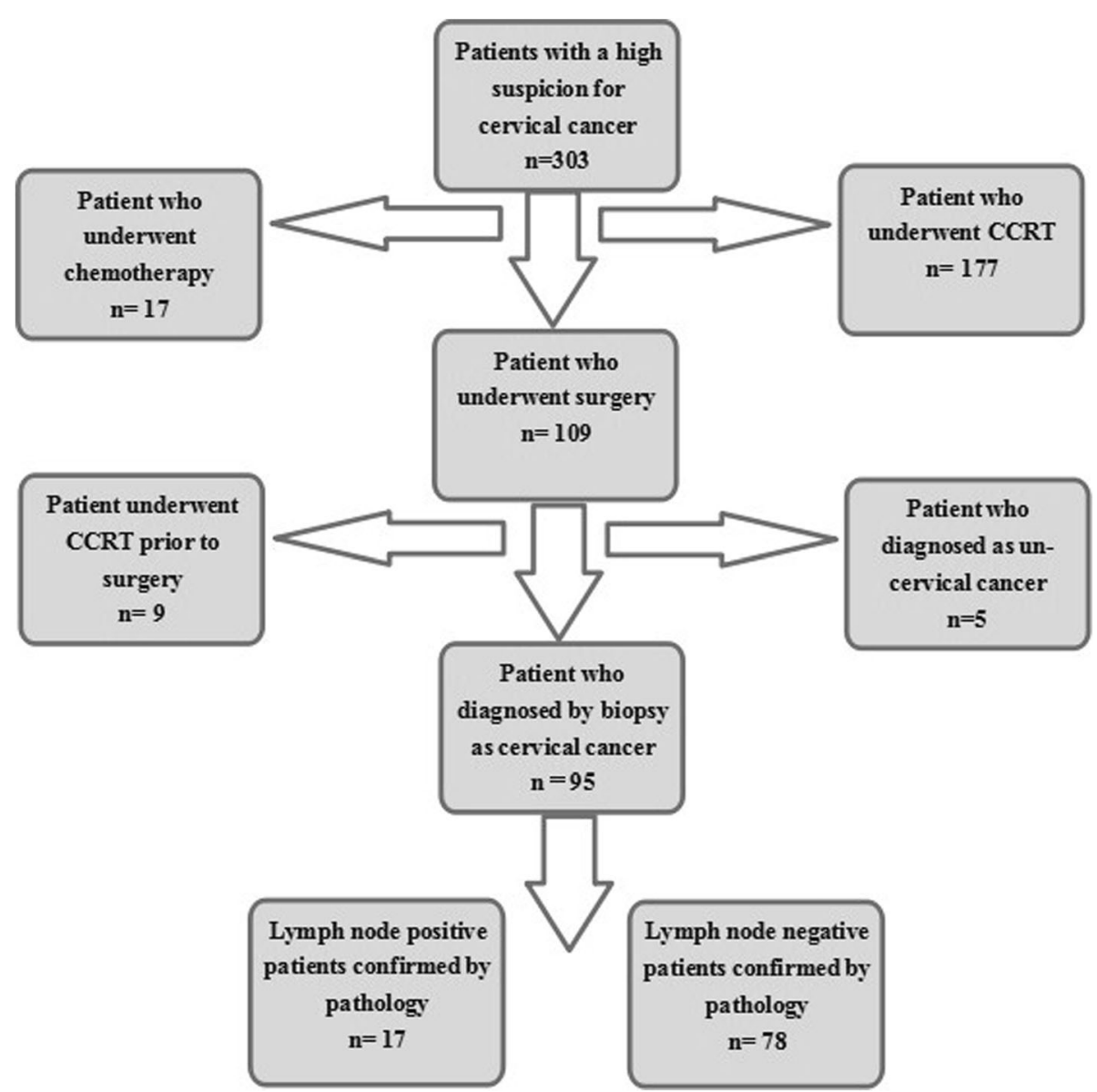

Fig. 2 Flowchart of patients who were referred to the assessment 

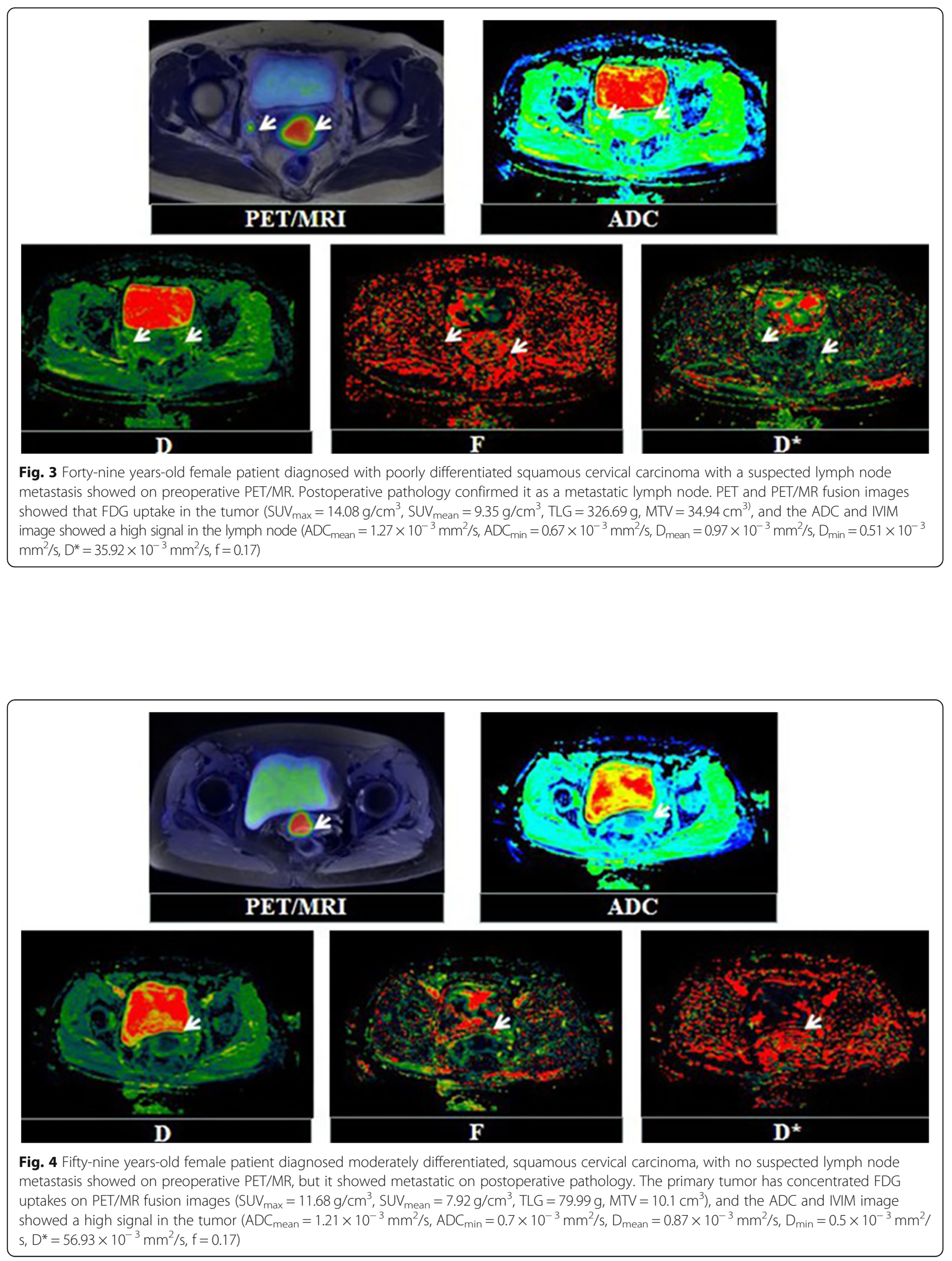
Table 2 Patients' clinicopathological characteristics

\begin{tabular}{ll}
\hline Clinical feature & Value \\
\hline No. of patients & 95 \\
Mean age (range) & 51.0 years (30-72) \\
FIGO stage (2018): & \\
Ib1 & $22(23.2 \%)$ \\
Ib2 & $33(34.7 \%)$ \\
Ib3 & $11(11.6 \%)$ \\
Ila1 & $4(4.2 \%)$ \\
Ila2 & $4(4.2 \%)$ \\
Ilb & $4(4.2 \%)$ \\
III & $17(17.9 \%)$ \\
Differentiation grade: & \\
Well differentiated & $19(20.0 \%)$ \\
Moderately differentiated & $63(66.3 \%)$ \\
Poorly differentiated & $13(13.7 \%)$ \\
Histologic type: & \\
Squamous carcinoma & $89(93.7 \%)$ \\
Adenocarcinoma & $6(6.3 \%)$ \\
Cervical stromal invasion depth: & \\
P $1 / 2$ & $36(37.9 \%)$ \\
Pathological diagnosis of lymph nodes: & $59(62.1 \%)$ \\
Negative & $17(17.9 \%)$ \\
\hline FIGO stage & $78(82.1 \%)$ \\
\hline
\end{tabular}

FIGO stage (2018):Postoperative pathological staging
Table 4 For all patients, univariate and multivariate analysis of parameters to predict lympha metastasis

\begin{tabular}{|c|c|c|c|c|}
\hline \multicolumn{5}{|c|}{$\begin{array}{l}\text { Method: Stepwise; Dependent Y: Lymphatic metastasis } \\
\text { Multivariate logistic regression: Enter Variable }<0.005\end{array}$} \\
\hline \multicolumn{5}{|c|}{ Univariate logistic regression analysis } \\
\hline Variable & coeffcients & Std Error & Wald & $P$ \\
\hline MTV & 0.065374 & 0.026612 & 6.0348 & 0.014 \\
\hline TLG & 0.0044791 & 0.0019118 & 5.4889 & 0.019 \\
\hline$D_{\min }$ & -4.41339 & 1.98372 & 4.9498 & 0.026 \\
\hline $\begin{array}{l}\text { PET in lymph node } \\
\text { diagnosis }\end{array}$ & 2.39356 & 0.60701 & 15.5488 & $<0.001$ \\
\hline $\begin{array}{l}\text { MRI in lymph node } \\
\text { diagnosis }\end{array}$ & 1.39544 & 0.55797 & 6.2547 & 0.0124 \\
\hline \multicolumn{5}{|c|}{ Multivariate logistic regression analysis } \\
\hline Variable & coeffcients & Std Error & Wald & $P$ \\
\hline TLG & 0.0083138 & 0.0028803 & 8.3317 & 0.0039 \\
\hline$D_{\min }$ & -10.14394 & 3.37033 & 9.0588 & 0.0026 \\
\hline $\begin{array}{l}\text { PET in lymph node } \\
\text { diagnosis }\end{array}$ & 4.45475 & 1.10161 & 16.3528 & 0.0001 \\
\hline
\end{tabular}

Univariate logistic analysis showed that MTV $(p=$ $0.014)$, TLG $(p=0.019), \mathrm{D}_{\min }(p=0.026)$, MRI for lymph node diagnosis $(p=0.012)$ and PET for lymph node diagnosis $(p<0.001)$ were associated with lymph node metastasis. Bonferroni corrected alpha value of 0.005 per test $(0.05 / 10)$ was applied to the final multivariate analyses in exploring lymph node metastasis. The combination of TLG, $\mathrm{D}_{\mathrm{min}}$, and PET for lymph node diagnosis had the strongest predictive value on multivariate logistic analysis (Table 4). The area under the ROC curve for

Table 3 The relationship between lymph node metastasis and imaging parameters in all patients

\begin{tabular}{|c|c|c|c|}
\hline \multirow[t]{2}{*}{ N } & Negative & Positive & \multirow[t]{2}{*}{$P$} \\
\hline & 78 & 17 & \\
\hline$D_{\text {mean }}\left(10^{-3} \mathrm{~mm}^{2} / \mathrm{s}\right)$ & $0.90 \pm 0.24$ & $0.81 \pm 0.16$ & 0.131 \\
\hline$D_{\min }\left(10^{-3} \mathrm{~mm}^{2} / \mathrm{s}\right)$ & $0.59 \pm 0.18$ & $0.49 \pm 0.13$ & 0.020 \\
\hline$A D C_{\text {mean }}\left(10^{-3} \mathrm{~mm}^{2} / \mathrm{s}\right)$ & $1.13 \pm 0.23$ & $1.16 \pm 0.26$ & 0.988 \\
\hline$A D C_{\min }\left(10^{-3} \mathrm{~mm}^{2} / \mathrm{s}\right)$ & $0.70 \pm 0.14$ & $0.76 \pm 0.22$ & 0.446 \\
\hline $\mathrm{D}^{*}\left(10^{-3} \mathrm{~mm}^{2} / \mathrm{s}\right)$ & $42.58 \pm 15.35$ & $42.87 \pm 17.70$ & 0.820 \\
\hline f & $0.16 \pm 0.06$ & $0.18 \pm 0.07$ & 0.738 \\
\hline$S U V_{\max }\left(\mathrm{g} / \mathrm{cm}^{3}\right)$ & $15.35 \pm 9.53$ & $19.24 \pm 8.69$ & 0.048 \\
\hline$S U V_{\text {mean }}\left(\mathrm{g} / \mathrm{cm}^{3}\right)$ & $9.16 \pm 6.03$ & $11.38 \pm 5.32$ & 0.061 \\
\hline $\operatorname{MTV}\left(\mathrm{cm}^{3}\right)$ & $10.22 \pm 8.06$ & $16.67 \pm 12.99$ & 0.081 \\
\hline TLG (g) & $100.11 \pm 117.33$ & $183.74 \pm 144.08$ & 0.014 \\
\hline \multicolumn{4}{|l|}{ PET in lymph node diagnosis } \\
\hline PET negative lymph nodes & 69 & 7 & \multirow[t]{2}{*}{$<0.001$} \\
\hline PET positive lymph nodes & 9 & 10 & \\
\hline \multicolumn{4}{|l|}{ MRI in lymph node diagnosis } \\
\hline MRI negative lymph nodes & 61 & 8 & \multirow[t]{2}{*}{0.009} \\
\hline MRI positive lymph nodes & 17 & 9 & \\
\hline
\end{tabular}



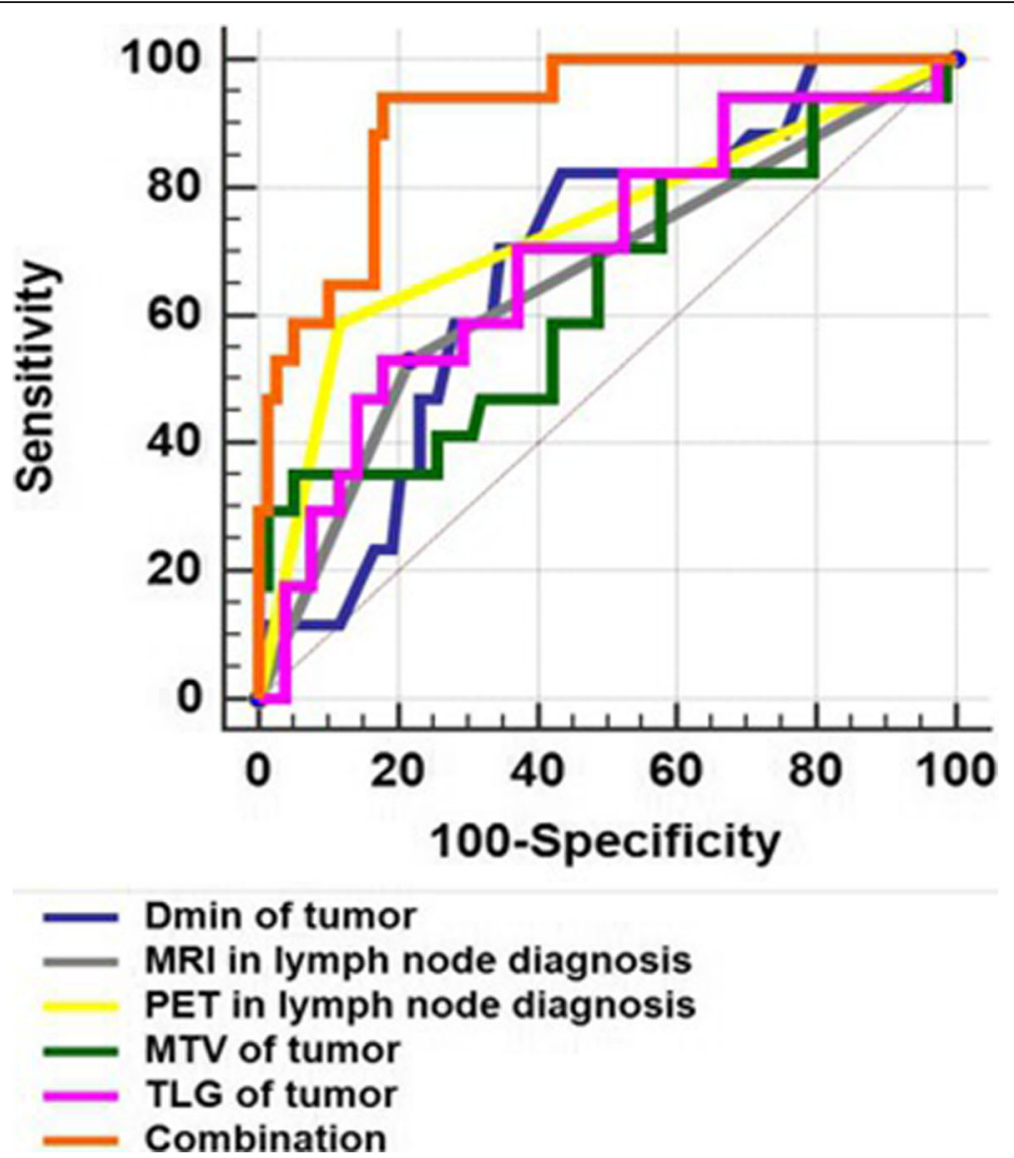

Fig. 5 For all patients, ROC analysis shows that $S U V_{\max }$ (AUC 0.654, 95\% confidence interval (CI) 0.549-0.749, $p=0.023$ ), SUV mean (AUC 0.646, 95\% Cl 0.541-0.741, $p=0.030$ ), TLG (AUC 0.692, 95\% Cl 0.588-0.782, $p=0.009$ ), $D_{\min }$ (AUC 0.681, 95\% Cl 0.577-0.773, $p=0.007$ ), MRI in lymph node diagnosis (AUC $0.656,95 \%$ Cl $0.551-0.750$, sensitivity 58.94, specificity $78.21, p=0.020$ ) and PET in lymph node diagnosis (AUC $0.736,95 \% \mathrm{Cl}$ $0.636-0.822$, sensitivity 58.82 , specificity $88.46, p<0.001$ ) had a positive effect on predicting metastatic lymph nodes confirmed by postoperative pathology. The area under the ROC curve for the combination of TLG, $D_{\min }$ and PET/MRI in lymph node diagnosis (AUC 0.913, 95\% Cl 0.8370.961 , sensitivity 94.12 , specificity $82.05, p<0.001$ ) was higher than any individual parameter (both, $p<0.05)$. Cl: confidence interval

Table 5 Spearman rank correlation coefficients between parameters

\begin{tabular}{|c|c|c|c|c|c|c|c|c|c|c|}
\hline & $S U V_{\max }$ & SUV $V_{\text {mean }}$ & TLG & MTV & $\mathrm{ADC}_{\text {mean }}$ & $A D C_{\text {min }}$ & $\mathrm{D}_{\text {mean }}$ & $D_{\text {min }}$ & $f$ & $D^{*}$ \\
\hline$S U V_{\max }$ & $\ldots$ & $0.980^{* *}$ & $0.603^{* *}$ & 0.126 & -0.039 & -0.039 & -0.079 & $-0.257^{*}$ & $-0.312^{*}$ & -0.057 \\
\hline$S U V_{\text {mean }}$ & $\ldots$ & $\ldots$ & $0.621^{* *}$ & $0.135^{*}$ & -0.027 & -0.056 & -0.111 & -0.210 & $-0.332^{*}$ & -0.056 \\
\hline TLG & $\ldots$ & $\ldots$ & $\ldots$ & $0.839^{* *}$ & -0.187 & -0.239 & -0.126 & $-0.226^{*}$ & $-0.285^{*}$ & -0.056 \\
\hline MTV & $\ldots$ & $\ldots$ & $\ldots$ & $\ldots$ & $-0.239^{*}$ & $-0.320^{*}$ & -0.132 & $-0.243^{*}$ & -0.175 & -0.020 \\
\hline$A D C_{\text {mean }}$ & $\ldots$ & $\ldots$ & $\ldots$ & $\ldots$ & $\ldots$ & $0.633^{* *}$ & $0.807^{* *}$ & $0.471^{* *}$ & $0.585^{* *}$ & 0.089 \\
\hline$A D C_{\text {min }}$ & & $\ldots$ & $\ldots$ & $\ldots$ & & & $0.564^{* *}$ & $0.853^{* *}$ & $0.345^{* *}$ & 0.039 \\
\hline$D_{\text {mean }}$ & $\ldots$ & $\ldots$ & $\ldots$ & $\ldots$ & $\ldots$ & $\ldots$ & $\ldots$ & $0.683^{* *}$ & $0.464^{* *}$ & 0.037 \\
\hline $\mathrm{D}_{\min }$ & & $\ldots$ & $\ldots$ & $\ldots$ & & $\ldots$ & $\ldots$ & $\ldots$ & $0.235^{*}$ & 0.042 \\
\hline f & $\ldots$ & $\ldots$ & $\ldots$ & $\ldots$ & $\ldots$ & $\ldots$ & $\ldots$ & $\ldots$ & $\ldots$ & $0.207^{*}$ \\
\hline$D^{*}$ & $\ldots$ & $\ldots$ & $\ldots$ & $\ldots$ & $\ldots$ & $\ldots$ & $\ldots$ & $\ldots$ & $\ldots$ & $\ldots$ \\
\hline
\end{tabular}


the combination of TLG, $\mathrm{D}_{\text {min }}$, and PET for lymph node diagnosis (AUC 0.913, $p<0.001$ ) was higher than that of any individual parameter $(p<0.05)$ (Fig. 5). The correlations between parameters are shown in Table 5 .

\section{The relationship between lymph node metastasis and imaging parameters in patients with PET negative lymph nodes}

A total of 76 patients were evaluated: 69 without lymph node metastasis and 7 with lymph node metastasis. $\mathrm{SUV}_{\max }$ (U test, $p=0.016$; AUC 0.797, $p<0.001$ ), SUVmean (U test, $p=0.018$; AUC 0.792, $\mathrm{p}<0.001$ ), MTV (U test, $p=0.013$; AUC 0.806, $p=0.024$ ), TLG (U test, $p=$ 0.004; AUC 0.855, $\mathrm{p}<0.001$ ), and $\mathrm{D}_{\min }$ (U test, $p=0.037$; AUC 0.758, $p=0.005$ ) showed significant between-group differences. The remaining parameters did not show statistical differences between the two groups. The TLG had the strongest predictive value according to the univariate and multivariate logistic analysis in the subset of patients with PET negative lymph nodes (Table 6).

\section{Discussion}

Recognition of lympha node metastasis before treatment in patients with cervical cancer is essential for personalized treatment plans, and diagnostic imaging is most commonly used for this purpose. In the present study, all data were measured by integrated PET-MRI. With the integrated device, a shortened scanning time, a reduced radiation dose, and the simultaneous PET and MRI parameters can be achieved, enabling combined multiparameter prediction and correlation [16-19].

The IVIM sequences was introduced into the present PET/MR study, which is an extension of diffusionweighted imaging (DWI) sequence, to PET/MRI. DWI reflects the diffusion dynamics of tissue water molecules by ADC; however, the attenuation of DWI signals in tissues is determined by both water molecule diffusion and microcirculation perfusion. ADC is a quantitative parameter based on single index model of DWI, and it

Table 6 For patients with PET negative lymph nodes, univariate and multivariate analysis of parameters to predict lympha node metastasis

Method: Stepwise; Dependent Y: Lymphatic metastasis Multivariate logistic regression: Enter Variable $<0.0167$

Univariate logistic regression analysis

\begin{tabular}{lllll} 
Variable & coeffcients & Std Error & Wald & $P$ \\
MTV & 0.13821 & 0.047032 & 8.6359 & 0.003 \\
TLG & 0.0084360 & 0.0029554 & 8.1477 & 0.004 \\
D min $_{\text {Multivariate logistic regression analysis }}$ & -7.32445 & 3.40917 & 4.6159 & 0.032 \\
Variable & coeffcients & Std Error & Wald & P \\
TLG & 0.014966 & 0.0056818 & 6.9379 & 0.0084 \\
\hline
\end{tabular}

reflects both the true diffusion of water molecules and the "false diffusion", caused by the blood microcirculation in the capillaries. This inability to reflect the movement of water molecules in living tissues limits the utility of DWI to evaluate microstructure changes.

The IVIM model compensates for these technical defects of traditional DWI. The theoretical basis is to doubleexponentially fit tissue signals based on multiple b-values, and quantitatively extract the main signal attenuation components from the low b-values of the curve. The true diffusion parameter value of the tissue was calculated at high bvalues [20]. So, this model has a higher accuracy to reflect the signal attenuation of water molecules than the DWI single exponential model. IVIM models can calculate three parameters, including $D$ (purediffusion coefficient), $f$ (perfusion fraction), and $\mathrm{D}^{*}$ (pseudo-diffusion coefficient). In the IVIM model, $\mathrm{D}$ and $\mathrm{f}$ value are indicators to reflect the true diffusion coefficient and perfusion information of the molecule, respectively. IVIM modalities have also been used in other cervical cancer studies [21, 22]. However, the current IVIM technology still has shortcomings and has not been applied on a large scale in the clinic setting. The limitations include: (1) The organs in the abdominal cavity may have a slight displacement movement during long-time scans, which may affect image post-processing; (2) image distortion caused by magnetic susceptibility artifacts at the gassoft tissue interface leads to artifacts obscuring lesions or unclear display of lesions, affecting data measurement and (3) imprecise ROIs including normal tissues result in poor measurement repeatability.

We established a combined prediction model for cervical cancer patients with lymphatic metastasis by multiple logistic regression analysis, using TLG and $D_{\min }$ of the tumor and PET for lymph node diagnosis while filtering out the remaining parameters. Several mechanisms may explain the strong predictive potential of these 3 combined parameters. In previous studies, lymph node with FDG uptake more than the background lymph node metabolism level was a diagnostic criterion for lymphatic metastasis $[8,9]$. Our study confirms the ability to diagnose lymphatic metastasis with this method (AUC 0.736, $p<0.001$ ), but also demonstrates its low sensitivity (58.82). In contrast, the combination of tumor TLG and $D_{\min }$ and this diagnostic criterion had better predictive value than the diagnostic criterion alone (AUC 0.913, sensitivity 94.12, specificity 82.05, $p<0.001$; difference between areas $0.176, \mathrm{Z}=3.141, p=$ $0.002)$. Previous studies have shown that the expression of glucose transporter-1 (Glut-1) is related to lymph node metastasis in a variety of tumors [23, 24], which will trigger an increase in FDG uptake and the corresponding quantitative indicators [25]. TLG is a comprehensive parameter that reflects the metabolic activity of the whole tumor. Compared with SUV $_{\max }$, TLG can 
assess tumor burden more accurately. A study involving patients with early cervical cancer showed that tumor MTV and TLG values are important imaging indicators for predicting lymphatic metastasis [26]. Similar conclusions have been obtained in studies involving endometrial carcinoma patients and thyroid carcinoma patients $[27,28]$.

Recently, many studies have quantitatively analyzed lymph nodes by IVIM to predict pelvic lymph node metastasis $[29,30]$. However, the slice of IVIM imaging is thick, which makes it difficult to carry out clinically concerning the usually small volume of lymph nodes. In this study, we evaluated lymphatic metastasis using IVIM parameters of tumors for cervical cancer such as the $\mathrm{D}$ value. Theoretically, the $\mathrm{D}$ value is not affected by the microcirculation blood flow of the tumor tissue, giving a more accurate signal on the diffusion of the tissue water molecules than does the ADC value. Studies have suggested that $\mathrm{D}$ values correlate with hypoxia and the tumor-stroma ratios $[18,31]$. $\mathrm{D}_{\min }$ value corresponds to the lowest tumor diffusion region, which may more accurately reflect the number and heterogeneity of tumor cells, as well as tumors with lymphatic metastasis.

A study using similar methods suggests that a combination of serum squamous cell carcinoma antigen (SCC$\mathrm{Ag}$ ) level, $\mathrm{SUV}_{\text {max }}$, and lymph node status is an important prognostic indicator for cervical cancer [32]. Another study suggested that the combination of lymph node CT indicators and tumor TLG values can improve the accuracy of PET/CT for the diagnosis of lymphatic metastasis in patients with cervical cancer [33]. Similar combined methods are also applied to breast cancer [34].

A previous study has shown that PET/MR for lymphatic metastasis was mainly dependent on the sensitivity of PET [35]. In this study, PET was also significantly better than MR in the diagnosis of lymph nodes. But we found a large number of PET false-negative cases, which accords with the findings of endometrial cancer and non-small cell lung cancer studies [36, 37]. Identifying PET false-negative cases has always been a clinical challenge. Therefore, we further explored the relationship between lymph node metastasis and imaging parameters in patients with PET/MRI-negative lymph nodes. By multivariate logistic analysis, TLG had the strongest predictive value for lymph node metastasis in this subset of patients.

This study has several limitations. On one hand, our scan range was from the vaginal level to the upper edge of the humerus. Therefore, we could not measure the $\mathrm{SUV}_{\text {peak }}$ value which may better reflect the aggressiveness of the tumor [25]. On the other hand, we used the pathologic diagnosis as the gold standard. The inclusion of patients who have undergone surgery results in fewer negative cases in the sampled population. A further evaluation of more cases is needed to verify our conclusions.

\section{Conclusions}

In all patients, the combination of tumor TLG and $D_{\min }$ of the tumor and PET for lymph node diagnosis had better predictive performance than the other imaging parameters for nodal metastasis. In patients with PET negative lymph nodes, TLG showed the strongest predictive potential. These findings may have clinical applications in personalized treatment planning for patients with cervical cancer.

\section{Abbreviations}

ADC: Apparent diffusion coefficient; CT: Computed tomography; D: Diffusionrelated coefficient; D*: Perfusion related diffusion coefficient; DWI: Diffusionweighted imaging; F: Perfusion-related parameter; FDG: Fluoro-D-glucose; FIGO: Federation International of Gynecology and Obstetrics; Glut-1: Glucose transporter-1; HE: Hematoxylin-eosin; IVIM: Intravoxel incoherent motion; MRI: Magnetic resonance imaging; MTV: Metabolic tumor volume; PET: Positron emission tomography; ROC: Receiver operating characteristic; ROI: Region of interest; SCC-Ag: Serum squamous cell carcinoma antigen; SUV $V_{\text {max: }}$ Maximum standardized uptake values; $S U V_{\text {mean }}$ : Mean standardized uptake values; TLG: Total lesion glycolysis

\section{Acknowledgements}

We would like to thank the native English-speaking scientists of BioMed Proofreading Company for editing our manuscript. Thanks Dr. Chengyan Dong from GE Healthcare for the revisions and language enhancement to our manuscript.

\section{Authors' contributions}

Conceptualization, Chen Xu, Xiaoran Li; Methodology, Chen Xu, Xiaoran Li; Formal Analysis, Chen Xu; Investigation, Chen Xu; Resources, Chen Xu; Data Curation, Yanchi Shi, Bo Wang; Writing-Original Draft Preparation, Chen Xu; Writing -Review \& Editing, Chen Xu; Supervision, Hongzan Sun; Project Administration, Hongzan Sun; Funding Acquisition, Hongzan Sun. All authors read and approved the final manuscript.

\section{Funding}

This study has received funding by National Natural Science Foundation of China (81401438), LIAONING Science \& Technology Project (2017225012), LIAONING Science Natural Science Foundation (2019-MS-373) and 345 Talent Project.

\section{Availability of data and materials}

The datasets used and/or analyzed during the current study are available from the corresponding author on reasonable request.

\section{Ethics approval and consent to participate}

Approved by the Shengjing hospital of China Medical University Technology ethics committees.

\section{Consent for publication \\ Publication is approved by all authors and tacitly or explicitly by the responsible authorities where the work was carried out.}

\section{Competing interests}

The authors declare that they have no competing interests.

Received: 25 October 2019 Accepted: 28 February 2020

Published online: 06 March 2020

\section{References}

1. Small W, Bacon MA, Bajaj A, Chuang LT, Fisher BJ, Harkenrider MM, et al. Cervical cancer: a global health crisis. Cancer. 2017;123(13):2404-12. 
2. Forouzanfar MH, Foreman KJ, Delossantos AM, Lozano R, Lopez AD, Murray CJ, et al. Breast and cervical cancer in 187 countries between 1980 and 2010: a systematic analysis. Lancet. 2011;378(9801):1461-84.

3. Shim SH, Lee SW, Park JY, Kim YS, Kim DY, Kim JH, et al. Risk assessment model for overall survival in patients with locally advanced cervical cancer treated with definitive concurrent chemoradiotherapy. Gynecol Oncol. 2013; 128(1):54-9.

4. Carlson RW, Scavone JL, Koh WJ, McClure JS, Greer BE, Kumar R, et al. NCCN framework for resource strati- fication: a framework for providing and improving global quality oncology care. J Compr Canc Netw. 2016;14(8):961-9.

5. Liu B, Gao S, Li S. A comprehensive comparison of CT, MRI, positron emission tomography or positron emission tomography/CT, and diffusion weighted imaging-MRI for detecting the lymph nodes metastases in patients with cervical cancer: a meta-analysis based on 67 studies. Gynecol Obstet Investig. 2017;82(3):209-22.

6. Grueneisen J, Schaarschmidt BM, Heubner M, Aktas B, Kinner S, Forsting M, et al. Integrated PET/MRI for whole-body staging of patients with primary cervical cancer: preliminary results. Eur J Nucl Med Mol Imaging. 2015; 42(14):1814-24.

7. Sarker A, Im HJ, Cheon GJ, Chung HH, Kang KW, Chung JK, et al. Prognostic implications of the SUV max $_{\text {max }}$ of primary tumors and metastatic lymph node measured by 18F-FDG PET in patients with uterine cervical cancer: a metaanalysis. Clin Nucl Med. 2016;41(1):34-40.

8. Onal C, Guler OC, Reyhan M, Yapar AF. Prognostic value of 18F-fluorodeoxyglucose uptake in pelvic lymph nodes in patients with cervical cancer treated with definitive chemoradiotherapy. Gynecol Oncol. 2015;137(1):40-6.

9. Kitajima K, Murakami K, Yamasaki E, Kaji Y, Sugimura K. Accuracy of integrated FDG-PET/contrast-enhanced $C T$ in detecting pelvic and paraaortic lymph node metastasis in patients with uterine cancer. Eur Radiol. 2009;19(6):1529-36.

10. Kidd EA, Thomas M, Siegel BA, Dehdashti F, Grigsby PW. Changes in cervical cancer FDG uptake during chemoradiation and association with response. Int J Radiat Oncol Biol Phys. 2013;85(1):116-22.

11. Akkas BE, Demirel BB, Dizman A, Vural GU. Do clinical characteristics and metabolic markers detected on positron emission tomography/ computerized tomography associate with persistent disease in patients with in-operable cervical cancer? Ann Nucl Med. 2013;27(8):756-63.

12. Le Bihan D, Breton $\mathrm{E}$, Lallemand $\mathrm{D}$, Aubin ML, Vignaud J, Laval-Jeantet M. Separation of diffusion and perfusion in intravoxel incoherent motion MRI imaging. Radiology. 1988;168(2):497-505.

13. Karsten B, Johannes G, Verena R, Paul B, Bahriye A, Philipp H, et al. [18F] FDG PET/MRI vs. PET/CT for whole-body staging in patients with recurrent malignancies of the female pelvis: initial results. Eur J Nucl Med Mol Imaging. 2015;42(1):56-65.

14. Klerkx WM, Veldhuis WB, Spijkerboer AM, Van Den Bosch MA, Mali WP, Heintz AP, et al. The value of 3.0 tesla diffusion-weighted MRI for pelvic nodal staging in patients with early stage cervical cancer. Eur J Cancer. 2012:48(18):3414-21.

15. Park SO, Kim JK, Kim KA, Park BW, Kim N, Cho G, et al. Relative apparent diffusion coefficient: determination of reference site and validation of benefit for detecting metastatic lymph nodes in uterine cervical cancer. J Magn Reson Imaging. 2009;29(2):383-90.

16. Yoo HJ, Lee JS, Lee JM. Integrated whole body MR/PET: where are we? Korean J Radiol. 2015;16(1):32-49.

17. Xu C, Sun HZ, Du SY, Xin J. Early treatment response of patients undergoing concurrent chemoradiotherapy for cervical cancer: an evaluation of integrated multi-parameter PET-IVIM MR. Eur J Radiol. 2019;117:1-8.

18. Zhang LO, Sun HZ, Bai XX, Chen ZW, Lu ZM, Xin J, et al. Correlation between tumor glucose metabolism and multiparametric functional MRI (IVIM and R2*) metrics in cervical carcinoma: evidence from integrated 18FFDG PET/MR. J Magn Reson Imaging. 2019;49(6):1704-12.

19. Du SY, Sun HZ, Gao S, Xin J, Lu ZM, Chen ZW, et al. Relationship between 18F-FDG PET metabolic parameters and MRI intravoxel incoherent motion (IVIM) histogram parameters and their correlations with clinicopathological features of cervical cancer: evidence from integrated PET/MRI. Clin Radiol. 2019;74(3):178-86.

20. Liu C, Liang C, Liu ZY, Zhang SX, Huang B. Intravoxel incoherent motion (IVIM) in evaluation of breast lesions: comparison with conventional DWI. Eur J Radiol. 2013;82(12):e782-9.

21. van Baalen S, Froeling M, Asselman M, Klazen C, Jeltes C, van Dijk L, et al. Mono, bi- and tri-exponential diffusion MRI modelling for renal solid masses and comparison with histopathological findings. Cancer Imaging. 2018;18(1): 44.

22. Becker AS, Perucho JA, Wurnig MC, Boss A, Ghafoor S, Khong PL, et al. Assessment of cervical cancer with a parameter-free Intravoxel incoherent motion imaging algorithm. Korean J Radiol. 2017;18(3):510-8.

23. Yang J, Wen J, Tian T, Lu ZS, Wang Y, Wang ZK, et al. GLUT-1 overexpression as an unfavorable prognostic biomarker in patients with colorectal cancer. Oncotarget. 2016;8(7):11788-96.

24. Sharen GW, Peng YJ, Cheng HD, Liu Y, Shi YH, Zhao J. Prognostic value of GLUT-1 expression in pancreatic cancer: results from 538 patients. Oncotarget. 2017:8(12):19760-7.

25. Zhang L, Sun HZ, Du SY, Xu WN, Xin J, Guo QY. Evaluation of 18F-FDG PET/ $\mathrm{CT}$ parameters for reflection of aggressiveness and prediction of prognosis in early-stage cervical cancer. Nucl Med Commun. 2018;39(11):1045-52.

26. Crivellaro C, Signorelli M, Guerra L, De Ponti E, Buda A, Dolci C, et al. 18FFDG PET/CT can predict nodal metastases but not recurrence in early stage uterine cervical cancer. Gynecol Oncol. 2012;127(1):131-5.

27. Husby JA, Reitan BC, Biermann M, Trovik J, Bjørge L, Magnussen IJ, et al. Metabolic tumor volume on 18F-FDG PET/CT improves preoperative identification of high-risk endometrial carcinoma patients. J Nucl Med. 2015; 56(8):1191-8.

28. Kim BH, Kim SJ, Kim K, Kim H, Kim SJ, Kim WJ, et al. High metabolic tumor volume and total lesion glycolysis are associated with lateral lymph node metastasis in patients with incidentally detected thyroid carcinoma. Ann Nucl Med. 2015;29(8):721-9.

29. Hauser T, Essig M, Jensen A, Laun FB, FBA M. Prediction of treatment response in head and neck carcinomas using IVIM-DWl: evaluation of lymph node metastasis. Eur J Radiol. 2014;83(5):783-7.

30. Markus S, Christiane K, Michael K, Azien Q, Maxim A, Cyrus B, et al. Preoperative evaluation of pelvine lymph node metastasis in high risk prostate cancer with intravoxel incoherent motion (IVIM) MRI. Eur J Radiol. 2018;107:1-6.

31. Li X, Wang P, Li D, Zhu H, Meng L, Song Y, et al. Intravoxel incoherent motion MR imaging of early cervical carcinoma: correlation between imaging parameters and tumor-stroma ratio. Eur Radiol. 2018;28(5):1875-83.

32. Nakamura K, Okumura Y, Kodama J, Hongo A, Kanazawa S, Hiramatsu Y. The predictive value of measurement of SUV $\max$ and SCC-antigen in patients with pretreatment of primary squamous cellcarcinoma of cervix. Gynecol. Oncol. 2010;119(1):81-6.

33. Li KX, Sun HZ, Guo QY. Combinative evaluation of primary tumor and lymph nodes in predicting pelvic lymphatic metastasis in early-stage cervical cancer: Amultiparametric PET-CT study. Eur J Radiol. 2019;113:153-7.

34. Song BI, Kim HW, Won KS. Predictive value of 18F-FDG PET/CT for axillary lymph node metastasis in invasive ductal breast cancer. Ann Surg Oncol. 2017;24(8):2174-81.

35. Heusch P, Buchbender C, Kohler J, Nensa F, Gauler T, Gomez B, et al. Thoracic staging in lung cancer: prospectivecomparison of 18F-FDG PET/MR imaging and 18F-FDG PET/CT. J Nucl Med. 2014;55(3):373-8.

36. Stewart Kl, Chasen B, Erwin W, Fleming N, Westin SN, Dioun S, et al. Preoperative PET/CT does not accurately detect extrauterine disease in patients with newly diagnosed high-risk endometrial cancer: a prospective study. Cancer. 2019;125(19):3347-53.

37. Taira N, Atsumi E, Nakachi S, Takamatsu R, Yohena T, Kawasaki H, et al. Comparison of GLUT-1, SGLT-1, and SGLT-2 expression in false-negative and true-positive lymph nodes during the F-FDG PET/CT mediastinal nodal staging of non-small cell lung cancer. Lung Cancer. 2018;09:123.

\section{Publisher's Note}

Springer Nature remains neutral with regard to jurisdictional claims in published maps and institutional affiliations. 